\title{
Fast and Flexible GPU Accelerated Binding Free Energy Calculations within the AMBER Molecular
} Dynamics Package

\author{
Daniel J. Mermelstein ${ }^{1}$, Charles Lin ${ }^{1,2}$, Gard Nelson ${ }^{3}$, Rachael Kretsch ${ }^{1,4}$, J. Andrew McCammon ${ }^{1}$, Ross C. \\ Walker $^{1,2, *}$
}

\author{
${ }^{1}$ Department of Chemistry \& Biochemistry, University of California San Diego, 9500 Gilman Drive, La \\ Jolla, CA, 92093, USA. \\ ${ }^{2}$ GlaxoSmithKline PLC, 1250 S. Collegeville Rd, Collegeville, PA, 19426, USA. \\ ${ }^{3}$ NantBioscience Inc, 9920 Jefferson Boulevard, Culver City, CA, 90232, USA \\ ${ }^{4}$ Harvey Mudd College, 301 Platt Blvd, Claremont, CA, 91711, USA \\ * Corresponding author: ross@rosswalker.co.uk
}

\begin{abstract}
:
Alchemical free energy calculations (AFE) based on molecular dynamics (MD) simulations are key tools in both improving our understanding of a wide variety of biological processes and accelerating the design and optimization of therapeutics for numerous diseases. Computing power and theory have, however, long been insufficient to enable AFE calculations to be routinely applied in early stage drug discovery. One of the major difficulties in performing AFE calculations is the length of time required for calculations to converge to an ensemble average. CPU implementations of MD based free energy algorithms can effectively only reach tens of nanoseconds per day for systems on the order of 50,000 atoms, even running on massively parallel supercomputers. Therefore, converged free energy calculations on large numbers of potential lead compounds are often untenable, preventing researchers from gaining crucial insight into molecular recognition, potential druggability, and other crucial areas of interest. Graphics Processing Units (GPUs) can help address this. We present here a seamless GPU implementation, within the PMEMD module of the AMBER molecular dynamics package, of thermodynamic integration (TI) capable of reaching speeds of $>140 \mathrm{~ns} /$ day for a 44,907-atom system, with accuracy equivalent to the existing CPU implementation in AMBER. The implementation described here is currently part of the AMBER 18 beta code and will be an integral part of the upcoming version 18 release of AMBER.
\end{abstract}

Keywords: Binding free energy, Molecular dynamics, AMBER, GPU accelerated, Drug Design,

\section{Introduction}

Computational chemists have for some time been seeking to correctly and universally predict the answer to the question "How well will ligand X bind to protein Y?" Couched in this is the implicit assumption that our predictive tool will be able to answer this question in a reasonable amount of time. For years, computational chemists have been forced to strike a difficult balance between speed and accuracy, often being forced to rely on faster, less accurate methods because (TI) and related Free Energy 
Perturbation (FEP) methods were simply too computationally intensive. However, the ceiling on the applicability of docking and MM/PBSA for use in making quantitative predictions of ligand binding, possibly the most relevant aspect of free energy in the pharmaceutical industry, has quickly become apparent. ${ }^{1}$ This has led to a renewed interest in improving slower but potentially more accurate methods such as TI. One of the primary difficulties which $\mathrm{TI}$ suffers from is a severe sampling limitation. ${ }^{2}$ This limitation is the result of several factors including the difficulty in sampling the relevant states, ${ }^{3}$ potentially dozens of unphysical intermediate states that must be simulated even for systems as small as host-guest systems, ${ }^{4}$ and the complexity and cost of the underlying molecular dynamics based algorithm. Thus, either hundreds of CPU cores and/or weeks of simulation time are required to obtain results, within acceptable error limits, for a single ligand-protein system in a drug design setting. This is untenable in an industrial setting where high-throughput is integral to success. The purpose of this work is to help address the throughput and finite sampling problem in TI using cost effective consumer hardware.

While there have been many different approaches to addressing the issue of sufficient sampling in $\mathrm{TI}$ calculations $\mathrm{s}^{5-7}$ the most straightforward manner of improving on the sampling is to write faster code. This paper details a substantially faster implementation of alchemical transformations using cost effective, NVIDIA GeForce graphics processors (GPUs). This code is fully implemented within the AMBER molecular dynamics package ${ }^{8-10}$ and takes direct advantage of the existing, highly efficient, GPU support. ${ }^{11-13}$ The implementation described here will be released as an integral part of the GPU accelerated PMEMD program in the upcoming AMBER 18 software release scheduled for Q2 2018. It is currently part of the AMBER 18 Beta code and a patch against AMBER 17 is available by request from the corresponding author.

\section{Theory and Methods}

\section{Model Calculations}

The goal of this paper is to demonstrate the efficacy and numerical precision of our new GPU TI implementation in AMBER. We have chosen two types of calculations for this purpose (Figure 1). The first, a free energy of solvation, the second, a binding affinity calculation.
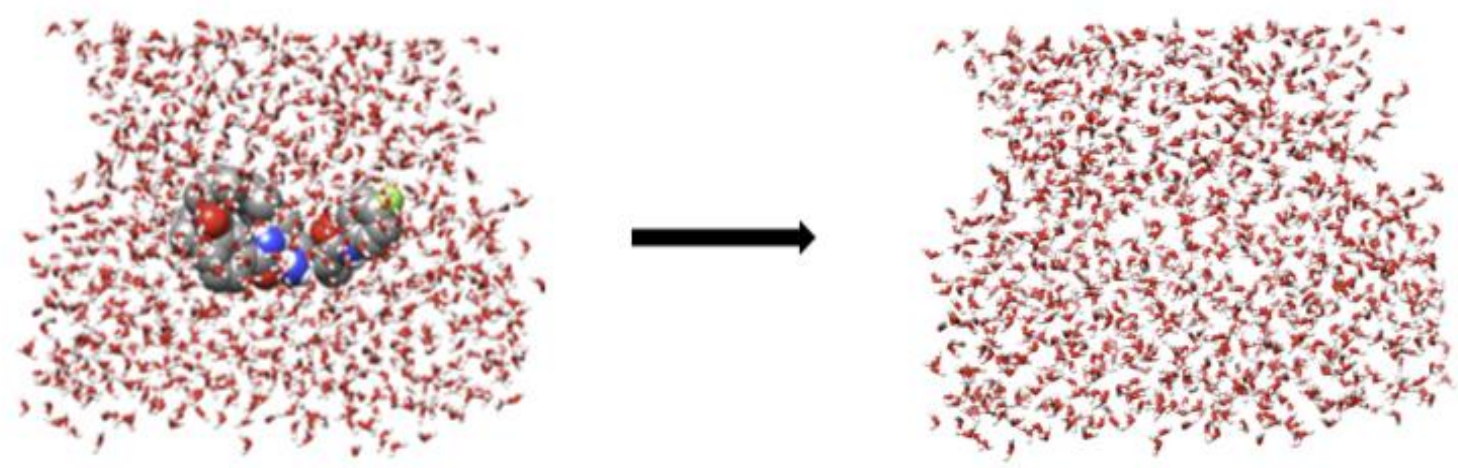

A. 


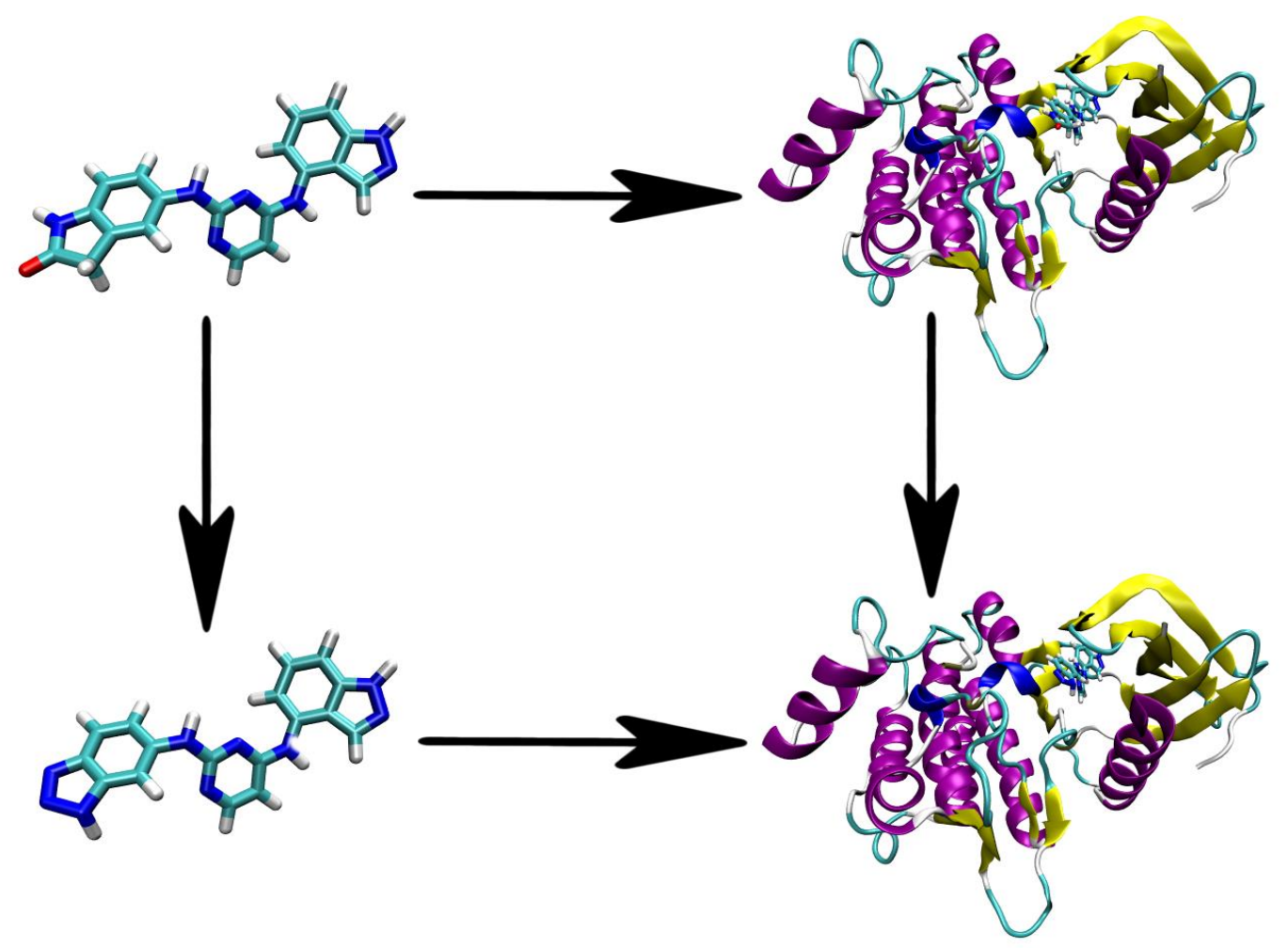

B.

Figure 1: Perturbation cycle for sample calculations performed to test the accuracy and performance of our new GPU implementation of TI. Each molecule represents a thermodynamic endpoint of a calculation. Top: Solvation free energy of N1((2S,3S,5R)-3-AMINO-6-(4-FLUOROPHENYLAMINO)- 5-METHYL-6-OXO-1-PHENYLHEXAN-2-YL)-N3,N3-

DIPROPYLISOPHTHALAMIDE, a promising BACE-1 inhibitor. ${ }^{14}$ Bottom: Relative free energy of binding of ligands GTC000107A and GTC000112A to spleen tyrosine kinase (Syk) from the GSK Syk database available through the D3R project. ${ }^{15}$ The transformation includes 3 deletions and two atom changes from GTC000107A to GTC000112A.

\section{Solvation free energy:}

For our present purpose of demonstrating the precision of our GPU TI code compared to the existing CPU based TI implementation it was important to test calculations that were difficult to converge. In the original implementation of $\mathrm{TI}$ in pmemd, ${ }^{16}$ the solvation free energy calculation was the most difficult to converge, despite free energies of solvation $\left(\Delta G_{\text {solv }}\right)$ having been one of the first types of free energy calculations attempted. ${ }^{17}$ In that instance, $\Delta \mathrm{G}_{\text {solv }}$ was defined as decoupling the ligand from the water over the course of one simulation (Figure 1A). We chose a potential inhibitor of beta secretase-1 (BACE-1), N1-((2S,3S,5R)-3-AMINO-6-(4-FLUOROPHENYLAMINO)- 5-METHYL-6-OXO-1PHENYLHEXAN-2-YL)-N3,N3- DIPROPYLISOPHTHALAMIDE as our test system here. ${ }^{18}$ 


\section{Relative free energy of binding}

Relative free energy of binding (RFEB) calculations are the most widely used application of thermodynamic integration given their utility in early stage (lead discovery and lead optimization) small molecule drug discovery. ${ }^{19,20}$ For this very reason we have chosen to demonstrate an RFEB calculation in this paper. Our example thermodynamic cycle for RFEB is shown in figure 1B for the spleen tyrosine kinase (Syk) system. Syk was chosen because it has a stable, well-defined binding pocket with a crystal structure. Ligands GTC000107A and GTC000112A were chosen from the D3R database because they were shown to have similar binding poses ${ }^{15}$ These similar binding poses meant that convergence issues were less likely to dominate the results allowing us to better test the GPU implementation and to compare the differences in the precision model between the CPU and GPU implementations. As is typical in RFEB calculations, alchemical transformations from GTC000107A to GTC000112A were performed for the ligands bound to the protein and the ligands free in solution.

\section{Docking of ligands to Syk}

Ligands were docked to crystal structure $1 X \mathrm{BA}^{21}$ using AutoDock Vina. ${ }^{22} \mathrm{Gaps}$ in the crystal structure (residues 360-362, 393-394 and 405-406) were filled using Modeller 9v2. ${ }^{23}$ Side chains for residues 402,420 , and 448 were treated as flexible, while the rest of the protein was treated as rigid. Ligands were fully flexible.

\section{Simulation details:}

All the simulations follow a similar protocol; any details that are specific to a particular system will be described below

\section{System preparation and equilibration}

The leap module in AMBER 16 was used to parametrize all systems. Protein models used the Amber ff14SB forcefield, ${ }^{21}$ with theTIP3P ${ }^{22}$ model for water. Neutralizing counterions, sodium or chloride, were added to each system as needed using TIP3P ions with parameters from Joung and Cheatham. ${ }^{23,24}$ Ligands were parametrized using the second generation generalized Amber forcefield $\left(\right.$ GAFF2) ${ }^{25}$ for the bonded and van der Waals parameters. Partial charges for ligands were obtained using RESP $^{26}$ fitting for the electrostatic potentials, calculated using Gaussian ${ }^{27}$ at the Hartree - Fock/6-31G* level of theory. A cubic periodic box was used with a minimum distance of $15 \AA$ between any box edge and any solute atom. All systems were minimized for 1000 cycles of steepest descent followed by 1000 cycles of conjugate gradient. Solute atoms were restrained with a restraint weight of $10 \mathrm{kcal} /\left(\mathrm{mol}{ }^{*} \AA\right)^{2}$. Minimization was followed, for all values of $\lambda$, by 100 ps of heating at constant volume and then $1 \mathrm{~ns}$ of equilibration at constant pressure. Temperature was regulated via a Langevin thermostat set to a target temperature of $300 \mathrm{~K}$ and a collision frequency of $5.0 \mathrm{ps}^{-1}$. Pressure was regulated using a Monte Carlo barostat with a target pressure of $1.0 \mathrm{~atm}$ and pressure relaxation time of $2.0 \mathrm{ps}$. 


\section{Production}

All production simulations were run in the NPT ensemble with a Langevin thermostat set to 300 $\mathrm{K}$ with collision frequency of $5.0 \mathrm{ps}^{-1}$, and a Monte Carlo barostat at a target pressure of $1.0 \mathrm{~atm}$ and pressure relaxation time of 2.0 ps. The direct space cutoff was set to $10 \AA$ for both van der Waals and electrostatics. Long range electrostatics we handled via the Particle Mesh Ewald (PME) method ${ }^{28}$ with a FFT grid spacing of 1 point per angstrom. Both the solvation free energy calculation and the RFEB calculation were run with 11 equally spaced lambda windows ranging from 0 to 1 . The default values for scalpha (0.5) and scbeta (12.0) were used. Energies were printed every $0.5 \mathrm{ps.} \mathrm{CPU} \mathrm{simulations} \mathrm{were} \mathrm{run}$ using pmemd.MPI from AMBER 16 with 12 Xeon Phi cores with a single socket running at $2.50 \mathrm{GHz}$ on an Intel Haswell standard compute node on XSEDE Comet. ${ }^{29}$ Pmemd.MPI was compiled with MVAPICH2 2.1. GPU simulations were run on NVIDIA GeForce Titan-X GPUs with Pascal architecture using the current AMBER 18 development tree with our GPU TI support incorporated. The GPU code was compiled for the default SPFP precision model using CUDA 8.0 and NVIDIA driver version 367.57. Both pmemd.MPI and pmemd.cuda were compiled using the gnu compiler in gnutools 2.69. Simulations were run with a $1 \mathrm{fs}$ time step (except for timing comparisons using $\mathrm{H}$-mass repartitioning). The solvation free energy system was simulated for $15 \mathrm{~ns}$. The RFEB complex and solvated systems were simulated for 10 ns. The first 5 ns of each simulation was discarded for equilibration purposes. The VDW change of the RFEB complex system required an additional $10 \mathrm{~ns}$ of simulation to converge. Each simulation was replicated three times with unique random seeds in each case.

\section{Single step alchemical change versus separate charge/NDW changes}

In transformations involving charge changes, it is possible in AMBER to use a softcore coulomb potential alongside the softcore Lennard-jones potential. To test both approaches for reproducibility between the two codes, the solvation free energy calculation was run with softcore electrostatics, while the RFEB was run in separate steps, turning charges off linearly before performing VDW changes.

\section{H-Mass Repartitioning}

Hydrogen masses bound to heavy atoms were repartitioned to 3.024 Daltons using ParmEd to allow for a 4 -fs time step. ${ }^{30}$ Some short simulations were run with identical input parameters, except the additional use of SHAKE to restrain the bonds between hydrogen and heavy atoms. To obtain the same number of data points, print and write frequencies were quadrupled for these simulations.

\section{Performance comparison between CPU and GPU}

All CPU simulations were run on using the pmemd.MPI module 12 Intel Xeon E5-2680v3 cores at $2.50 \mathrm{GHz}$ on a single socket of an Intel Haswell standard compute node on XSEDE Comet. All GPU simulations were run on NVIDIA GeForce Titan-X (Pascal) GPUs with driver 367.57 and CUDA 8.0. 


\section{Analysis}

All integrations were carried out using a cubic spline over 11 lambda windows: 0.0, 0.1, 0.2, 0.3, $0.4,0.5,0.6,0.7,0.8,0.9,1.0$. The Alchemical analysis python package ${ }^{31}$ was used to calculate our free energy estimates and associated errors. Autocorrelation times were estimated using pymbar ${ }^{32}$. Uncertainties for average values are the standard deviation of the replicate calculations.

\section{Results and Discussion}

\section{Numerical comparison}

Previous work by several groups has shown the CPU implementation of TI to be already capable of predicting experimental free energies. ${ }^{33-36}$ Thus for the purposes of validating our GPU implementation we consider values that agree within statistical error to the CPU implementation to indicate success.

The results of the solvation free energy and RFEB calculations are shown below (Table 1). The average results of the CPU and GPU simulations agree to within the standard deviation of three replicates.

\begin{tabular}{|c|c|c|}
\hline calculation & code & $\begin{array}{c}\text { Relative Gibbs free energy change } \\
\text { (kcal/mol) }\end{array}$ \\
\hline Solvation free energy & CPU & $53.0 \pm 2.4$ \\
\hline & GPU & $53.8 \pm 2.4$ \\
\hline RFEB - complex & CPU & $27.50 \pm 0.46$ \\
\hline RFEB - solution & GPU & $27.36 \pm 0.55$ \\
\hline & CPU & $28.95 \pm 0.12$ \\
\hline RFEB $(\Delta \Delta G)$ & GPU & $28.86 \pm 0.05$ \\
\hline & CPU & $-1.45 \pm 0.48$ \\
\hline
\end{tabular}

Table 1: Numerical comparison of free energy of solvation and RFEB calculations on CPU and GPU. All values are the average \pm the standard deviation of three replicates. RFEB - complex is the sum of the simulations in which the ligands are bound to the protein. RFEB - solution is the sum of the simulations in which the ligands are free in solution. Values for individual charge transformation and Lennard-Jones transformation simulation can be found in SI table 1.

The results agree between the CPU and GPU codes but, as shown with the performance numbers below, the GPU code obtained the result in approximately $1 / 30^{\text {th }}$ of the time it took to run the calculations on all CPUs cores within the node. For the RFEB calculation, the charge and VDW changes were carried out separately. These calculations also agreed to within statistical error (SI table 2).

Since the values measured here are ensemble averages using a Langevin thermostat that introduces random friction forces to control the temperature, it is still potentially possible that while the two codes agree within statistical error that we are using an incorrect potential in the GPU code due to some subtle implementation bug. It is thus useful, from a code validation perspective, to directly compare the potentials (both energy and gradients) between the CPU and GPU codes. Table $\mathbf{2}$ below compares initial energies for the same starting structure between the CPU TI (DPDP precision model) and GPU TI (SPFP precision model). 


\begin{tabular}{|c|c|c|}
\hline Term & CPU $(\mathrm{kcal} / \mathrm{mol})$ & GPU (kcal/mol) \\
\hline VDW non-bonded & 16209.8375 & 16209.8375 \\
\hline Electrostatic non-bonded & -171344.5200 & -171344.5191 \\
\hline Bonded terms & 19937.6161 & 19937.6162 \\
\hline DVDL & -35.4512 & -35.4518 \\
\hline
\end{tabular}

Table 2: Numerical comparison of energies at step 0 of our RFEB trajectories. Results are for Lennard-Jones parameter change with softcore VDW potential. Electrostatic non-bonded includes both direct space and PME reciprocal space terms. "Bonded terms" includes bonds, angles, dihedrals, and 1-4 adjustment terms. DVDL, the derivative of potential with respect to lambda, is the sum of the contributions from all potential terms (bonded, VDW, and electrostatic). Forces were also equivalent to the same numerical precision, but are not shown due to the sheer number of atoms for which force is calculated.

Energy and DVDL values agree to at least 6 significant figures which agrees with previous comparisons of conventional MD simulations between the DPDP (CPU) and SPFP (GPU) precision models. This slight difference is due to different charge representations between the CPU and GPU code. The CPU code misrepresents the charges beyond the $6^{\text {th }}$ decimal place on certain atoms.

\section{Performance comparison}

The use of GPUs has allowed for a greater than 30X average performance increase over a single socket 12 Intel Xeon E5-2680v3 core node. (Figure 2).

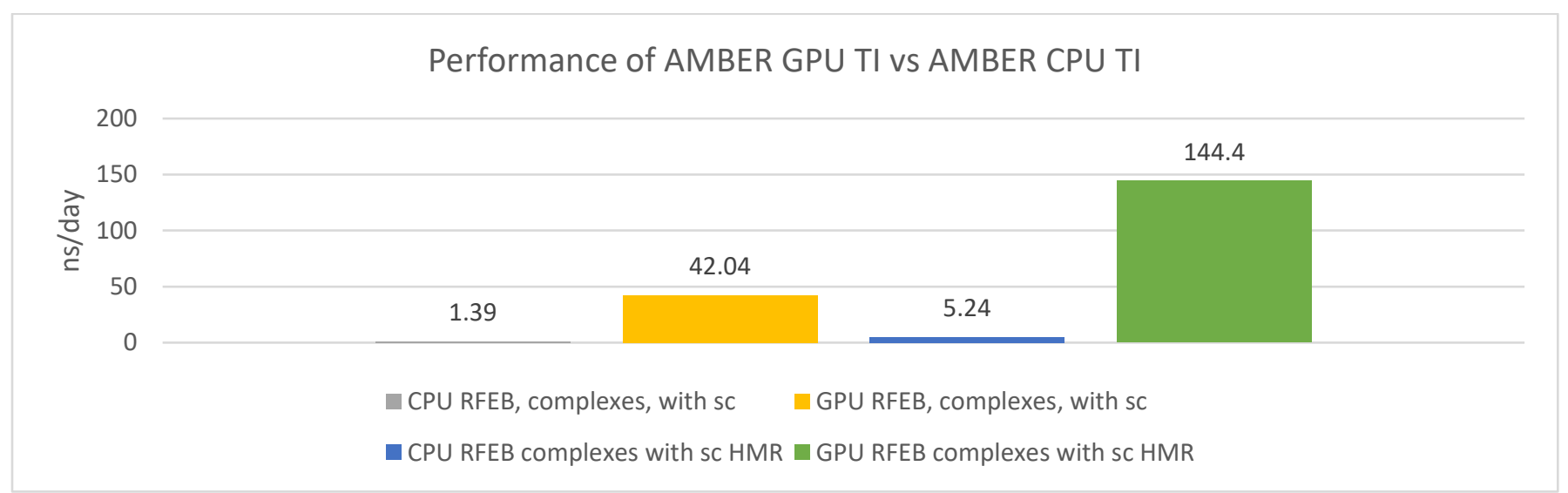

Figure 2: comparison of average performance of CPU and GPU TI code for a protein-ligand binding calculation. CPU code was run on 12 Intel Xeon E5-2680v3 cores, while the GPU code was run on a single NVIDIA GeForce Titan-X [Pascal] GPU. Numbers are from a VDW transformation from the lambda $=0$ window. There was a total of 44,907 atoms, 81 of which were defined as TI atoms, with 3 being softcore. Charges on the softcore atoms were turned off. The GPU code performance did not scale as well with HMR compared with the CPU code (3.43X vs 3.77X) because of the higher energy printing frequency (every 125 steps) which more negatively affects GPU performance than CPU performance.

Hydrogen mass repartitioning enabled the use of a 4-fs time step. We noticed no issues in using 4-fs as a time step, in keeping with work done by others. ${ }^{30,37}$ Overall, we have achieved a greater than 360X performance enhancement for RFEB protein-ligand complex systems over a single CPU core, or greater than 30X performance improvement over a CPU node. With our code, these calculations can now be carried out on the order of hours on a single cost-efficient GeForce GPU, instead of weeks on a single node or having to utilize large number of CPU nodes with expensive interconnects. For way of reference the node used in this work can be purchased for approximately $\$ 2800$ with a single GeForce Titan-X [Pascal] GPU. Our recommended configuration would, at the time of writing, be a system 
containing 2 x E5-2620V4 CPUs, 64GB of memory and 4 x NVIDIA GeForce 1080TI GPUs. This configuration currently costs between $\$ 5000$ and $\$ 6500$ and can run 4 lambda windows at once, one on each GPU, obtaining performance equivalent to that shown above for each window simultaneously.

\section{Conclusion}

We have demonstrated GPU enabled TI in the AMBER molecular dynamics suite that will form the officially supported GPU TI implementation to be released in the upcoming AMBER v18. Our implementation compared with the most recent version of $\mathrm{TI}$ in pmemd $\mathrm{d}^{8}$ is over $360 \mathrm{X}$ faster for proteinligand binding than a single CPU core, 30X faster compared with a single node if using a single GPU per node and $120 X$ faster per node if one considers that 4 GeForce GPUs can be added to a single node in a very cost effective manner. These performance improvements come without sacrificing precision or accuracy. The input format is identical to CPU pmemd, making the transition seamless for existing users. MBAR support has also been implemented and is in the process of being performance optimized. MBAR support will be available with the AMBER 18 release and the implementation will be discussed in follow up publications. The code described in this manuscript is available by request from the corresponding author as a patch against the release version of AMBER 17 and applicable updates as of Sept $1^{\text {st }}, 2017$.

\section{Acknowledgments}

The authors thank Woody Sherman of Silicon Therapeutics, Thomas Fox of BoehringerIngelheim and Sasha Buzkho of Nant Biosciences for extensively beta testing the software and providing critical feedback. Daniel Mermelstein is supported in part by the Interfaces training program for multi scale biology from the National Institutes of Health (NIH). Work in the JAM group is supported in part by $\mathrm{NIH}, \mathrm{NBCR}$, and the NSF supercomputer centers. RCW's contributions were funded by royalties received from licensing of the AMBER software by UCSF. No contributions to this work, monetary or otherwise, were made by NVIDIA Corp.

\section{$\underline{\text { References }}$}

1. Mobley, D. L. \& Dill, K. A. Binding of Small-Molecule Ligands to Proteins: 'What You See' Is Not Always 'What You Get'. Structure (2009). doi:10.1016/j.str.2009.02.010

2. Procacci, P. Alchemical determination of drug-receptor binding free energy: Where we stand and where we could move to. J. Mol. Graph. Model. 71, 233-241 (2017).

3. Chipot, C. \& Pohorille, A. SPRINGER SERIES IN CHEMICAL PHYSICS 86 Free Energy Calculations Theory and Applications in Chemistry and Biology. (2007).

4. Yin, J., Niel Henriksen, B. M., Chiu, M. W., David Mobley, B. L. \& Michael Gilson, B. K. Overview of the SAMPL5 host-guest challenge: Are we doing better? doi:10.1007/s10822-016-9974-4

5. Shirts, M. R. \& Pande, V. S. Solvation free energies of amino acid side chain analogs for common molecular mechanics water models. J. Chem. Phys. 122, (2005).

6. Bruckner, S. \& Boresch, S. Efficiency of alchemical free energy simulations. I. A practical comparison of the exponential formula, thermodynamic integration, and Bennett's acceptance 
ratio method. J. Comput. Chem. (2011). doi:10.1002/jcc.21713

7. Bruckner, S. \& Boresch, S. Efficiency of alchemical free energy simulations. II. Improvements for thermodynamic integration. J. Comput. Chem. (2011). doi:10.1002/jcc.21712

8. D.A. Case, D.S. Cerutti, T.E. Cheatham, III, T.A. Darden, R.E. Duke, T.J. Giese, H. Gohlke, A.W. Goetz, D. Greene, N. Homeyer, S. Izadi, A. Kovalenko, T.S. Lee, S. LeGrand, P. Li, C. Lin, J. Liu, T. Luchko, R. Luo, D. Mermelstein, K.M. Merz, G. Monard, H. Nguyen, I. Omelyan, A. Onufriev, F. Pan, R. Qi, D.R. Roe, A. Roitberg, C. Sagui, C.L. Simmerling, W.M. Botello-Smith, J. Swails, R.C. Walker, J. Wang, R.M. Wolf, X. Wu, L. Xiao, D.M. York and P.A. Kollman (2017), AMBER 2017, University of California, San Francisco.

9. D.A. Case, D.S. Cerutti, T.E. Cheatham, III, T.A. Darden, R.E. Duke, T.J. Giese, H. Gohlke, A.W. Goetz, D. Greene, N. Homeyer, S. Izadi, A. Kovalenko, T.S. Lee, S. LeGrand, P. Li, C. Lin, J. Liu, T. Luchko, R. Luo, D. Mermelstein, K.M. Merz, G. Monard, H. Nguyen, I. Omelyan, A. Onufriev, F. Pan, R. Qi, D.R. Roe, A. Roitberg, C. Sagui, C.L. Simmerling, W.M. Botello-Smith, J. Swails, R.C. Walker, J. Wang, R.M. Wolf, X. Wu, L. Xiao, D.M. York and P.A. Kollman (2016), AMBER 2016, University of California, San Francisco.

10. Salomon-Ferrer, R., Case, D. A. \& Walker, R. C. An overview of the Amber biomolecular simulation package. Wiley Interdiscip. Rev. Comput. Mol. Sci. 3, 198-210 (2013).

11. Götz, Andreas W.; Williamson, Mark J.; Xu, Dong; Poole, Duncan; Le Grand, Scott; Walker, Ross C. Routine microsecond molecular dynamics simulations with AMBER on GPUs. 1. generalized born. J. Chem. Theory Comput. 8, 1542-1555 (2012).

12. Salomon-Ferrer, R., Götz, A. W., Poole, D., Le Grand, S. \& Walker, R. C. Routine microsecond molecular dynamics simulations with AMBER on GPUs. 2. Explicit solvent particle mesh ewald. J. Chem. Theory Comput. 9, 3878-3888 (2013).

13. Le Grand, S., Götz, A. W. \& Walker, R. C. SPFP: Speed without compromise - A mixed precision model for GPU accelerated molecular dynamics simulations. Comput. Phys. Commun. 184, 374380 (2013).

14. Kim, M. O., Blachly, P. G. \& McCammon, J. A. Conformational Dynamics and Binding Free Energies of Inhibitors of BACE-1: From the Perspective of Protonation Equilibria. PLoS Comput. Biol. 11, 128 (2015).

15. GSK Syk Database. D3R Project (Accessed May 24, 2017) at <https://drugdesigndata.org/about/datasets/225>

16. Kaus, J. W., Pierce, L. T., Walker, R. C. \& McCammon, J. A. Improving the efficiency of free energy calculations in the amber molecular dynamics package. J. Chem. Theory Comput. (2013). doi:10.1021/ct400340s

17. Postma, J. P. M.; Berendsen, H. J. C.; Haak, J. R., Thermodynamics of cavity formation in water: a molecular dynamics study, F. S. C. S. Thermodynamics of cavity formation in water. A molecular dynamics study. Faraday Symp. Chem. Soc. 17, 55-67 (1982).

18. Yang, Wenjin; Lu, Wanli; Lu, Yafan; Zhong, Min; Sun, Jian; Thomas, Anila E.; Wilkinson, Jennifer M.; Fucini, Raymond V.; Lam, Melissa; Randal, Mike; Shi, Xiao Ping; Jacobs, Jeffrey W.; McDowell, Robert S.; Gordon, Eric M.; Ballinger, Marcus D. Aminoethylenes: A tetrahedral intermediate isostere yielding potent inhibitors of the aspartyl protease BACE-1. J. Med. Chem. 49, 839-842 
(2006).

19. Tembe, B. L. \& Mc Cammon, J. A. Ligand-receptor interactions. Comput. Chem. 8, 281-283 (1984).

20. Wereszczynski, J. \& McCammon, J. A. Statistical mechanics and molecular dynamics in evaluating thermodynamic properties of biomolecular recognition. Quarterly Reviews of Biophysics (2012). doi:10.1017/S0033583511000096

21. Maier, James A.; Martinez, Carmenza; Kasavajhala, Koushik; Wickstrom, Lauren; Hauser, Kevin E.; Simmerling, Carlos. ff14SB: Improving the Accuracy of Protein Side Chain and Backbone Parameters from ff99SB. J. Chem. Theory Comput. 11, 3696-3713 (2015).

22. Jorgensen, W. L., Chandrasekhar, J., Madura, J. D., Impey, R. W. \& Klein, M. L. Comparison of simple potential functions for simulating liquid water. J. Chem. Phys. 79, 926 (1983).

23. Joung, I. S. Halide lons Using Water-Model-Specific lon Parameters. 13279-13290 (2009).

24. Joung, I. S. \& Cheatham, T. E. Determination of alkali and halide monovalent ion parameters for use in explicitly solvated biomolecular simulations. J. Phys. Chem. B 112, 9020-9041 (2008).

25. Wang, J., Wolf, R. M., Caldwell, J. W., Kollman, P. A. \& Case, D. A. Development and Testing of a General Amber Force Field. J Comput Chem 25, 1157-1174 (2004).

26. Cornell, Wendy D.; Cieplak, Piotr; Bayly, Christopher I.; Gould, lan R.; Merz, Kenneth M.; Ferguson, David M.; Spellmeyer, David C.; Fox, Thomas; Caldwell, James W.; Kollman, Peter A. A second generation force field for the simulation of proteins, nucleic acids, and organic molecules. J. Am. Chem. Soc. 117, 5179-5197 (1995).

27. M. J. Frisch, G. W. Trucks, H. B. Schlegel, G. E. Scuseria, M. A. Robb, J. R. Cheeseman, G. Scalmani, V. Barone, B. Mennucci, G. A. Petersson, H. Nakatsuji, M. Caricato, X. Li, H. P. Hratchian, A. F. Izmaylov, J. Bloino, G. Zheng, J. L. Sonnenberg, M. Had, 2009. Gaussian 09 Revision E.01. (2009).

28. Darden, T., York, D. \& Pedersen, L. Particle mesh Ewald: An N·log(N) method for Ewald sums in large systems. J. Chem. Phys. 98, 10089 (1993).

29. Comet XSEDE User Guide. (Accessed May 24, 2017) at <https://portal.xsede.org/sdsc-comet>

30. Hopkins, C. W., Le Grand, S., Walker, R. C. \& Roitberg, A. E. Long-time-step molecular dynamics through hydrogen mass repartitioning. J. Chem. Theory Comput. (2015). doi:10.1021/ct5010406

31. Klimovich, P. V., Shirts, M. R. \& Mobley, D. L. Guidelines for the analysis of free energy calculations. J. Comput. Aided. Mol. Des. 397-411 (2015). doi:10.1007/s10822-015-9840-9

32. Chodera, J. D. A simple method for automated equilibration detection in molecular simulations. bioRxiv 21659 (2015). doi:10.1101/021659

33. Su, P. C. \& Johnson, M. E. Evaluating thermodynamic integration performance of the new amber molecular dynamics package and assess potential halogen bonds of enoyl-ACP reductase (Fabl) benzimidazole inhibitors. J. Comput. Chem. (2016). doi:10.1002/jcc.24274

34. Park, J., McDonald, J. J., Petter, R. C. \& Houk, K. N. Molecular Dynamics Analysis of Binding of Kinase Inhibitors to WT EGFR and the T790M Mutant. J. Chem. Theory Comput. (2016). doi:10.1021/acs.jctc.5b01221 
35. Ucisik, M. N. \& Hammes-Schiffer, S. Relative Binding Free Energies of Adenine and Guanine to Damaged and Undamaged DNA in Human DNA Polymerase ??: Clues for Fidelity and Overall Efficiency. J. Am. Chem. Soc. (2015). doi:10.1021/jacs.5b08451

36. Lee, H. C., Hsu, W. C., Liu, A. L., Hsu, C. J. \& Sun, Y. C. Using thermodynamic integration MD simulation to compute relative protein-ligand binding free energy of a GSK3?? kinase inhibitor and its analogs. J. Mol. Graph. Model. (2014). doi:10.1016/j.jmgm.2014.04.010

37. Henriksen, N. M., Fenley, A. T. \& Gilson, M. K. Computational Calorimetry: High-Precision Calculation of Host-Guest Binding Thermodynamics. J. Chem. Theory Comput. 11, 4377-4394 (2015). 


\section{Supporting info}

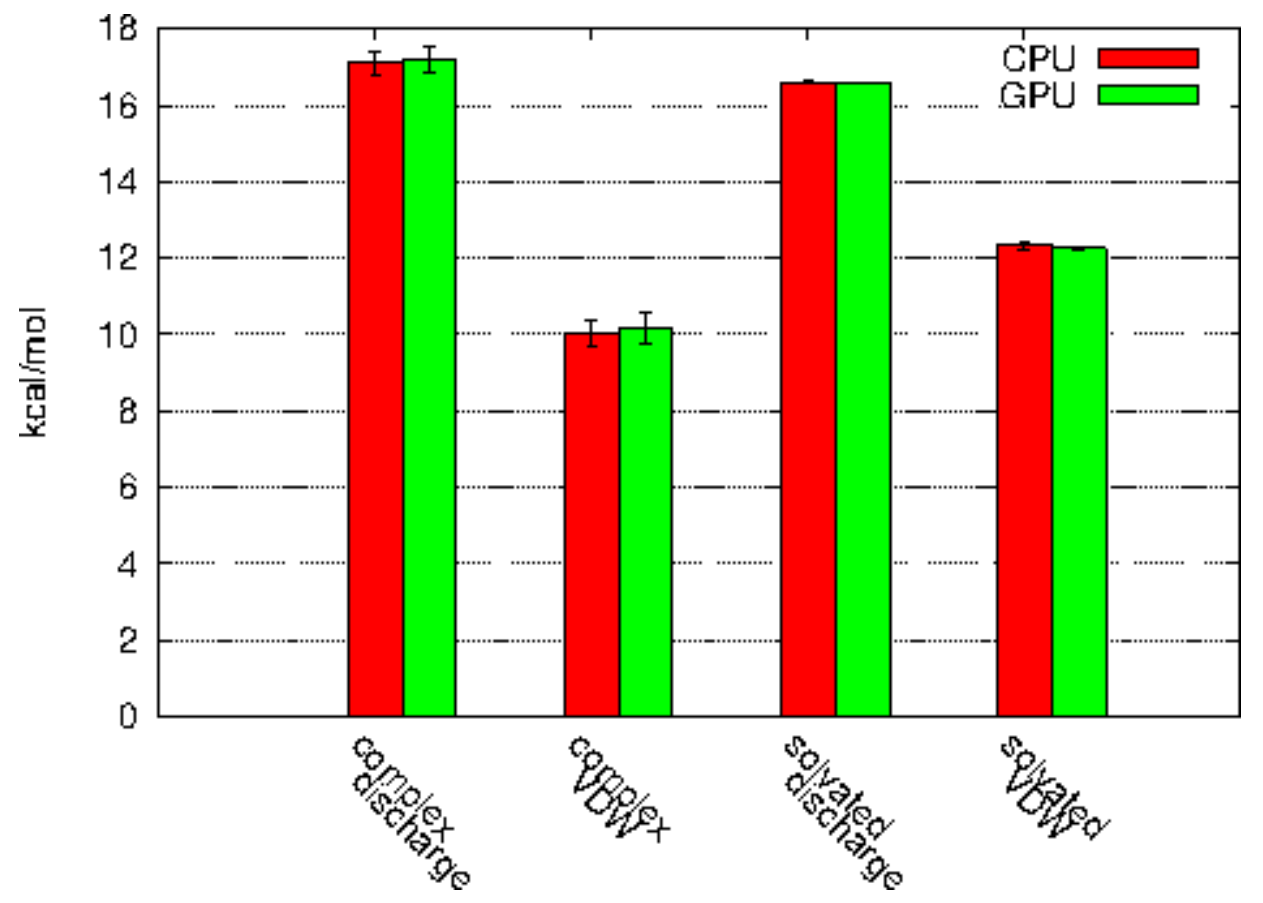

SI Figure 1: breakdown of RFEB calculation into charge change and Lennard Jones change. All caculated numbers agree within error (SI Table 1).

\begin{tabular}{|l|l|l|l|}
\hline \multicolumn{2}{|l|}{ calc } & step & Relative Gibbs free energy change (kcal/mol) \\
\hline \multirow{4}{*}{ ligand binding - complex } & cpu & discharge & $17.11 \pm 0.29$ \\
\cline { 2 - 4 } & gpu & discharge & $17.21 \pm 0.36$ \\
\cline { 2 - 4 } & cpu & vdw & $10.38 \pm 0.36$ \\
\cline { 2 - 4 } & gpu & vdw & $10.15 \pm 0.42$ \\
\hline \multirow{4}{*}{ ligand binding - ligands } & cpu & discharge & $16.61 \pm 0.01$ \\
\cline { 2 - 4 } & gpu & discharge & $16.59 \pm 0.02$ \\
\cline { 2 - 4 } & cpu & vdw & $12.34 \pm 0.11$ \\
\cline { 2 - 4 } & gpu & vdw & $12.27 \pm 0.03$ \\
\hline
\end{tabular}

SI Table 1: numerical values associated with SI figure 1. 
| TI region 1

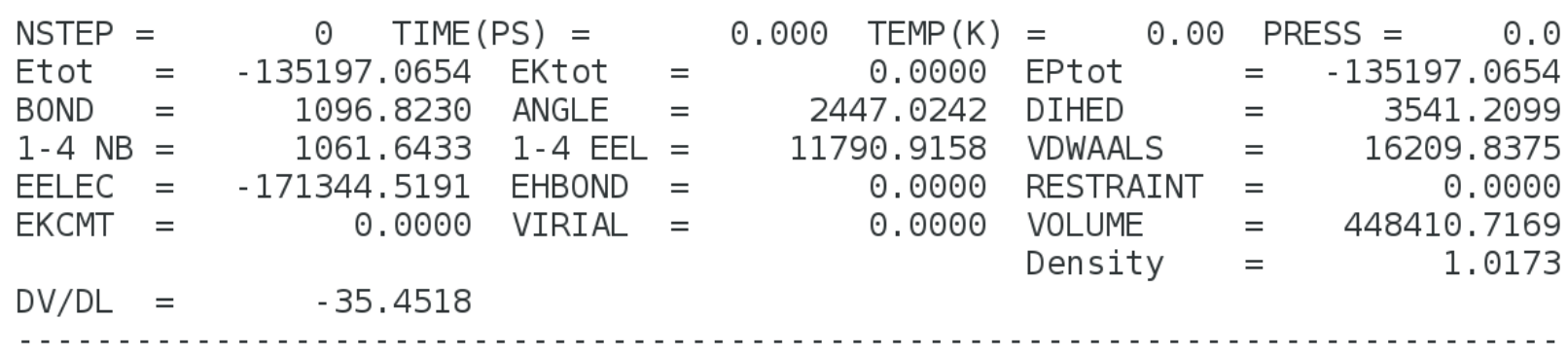

Softcore part of the system: 3 atoms, $\operatorname{TEMP}(\mathrm{K})=215.86$

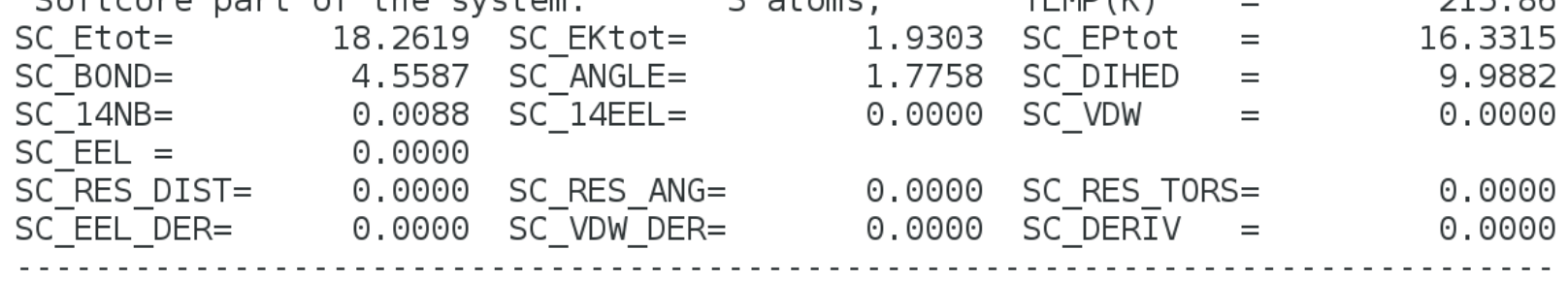

| TI region 2

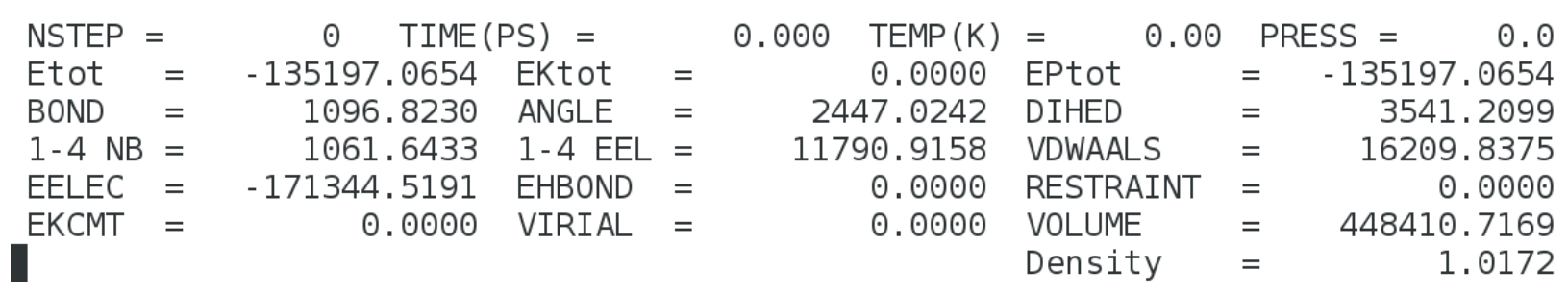

VDW GPU 
| TI region 1

\begin{tabular}{|c|c|c|c|c|c|c|c|c|c|}
\hline NSTEP & $=$ & TIME & S) & & 0.000 & $\operatorname{TEMP}(\mathrm{K})$ & 0.00 & \multicolumn{2}{|c|}{ PRESS = } \\
\hline Etot & $=$ & -135197.0665 & EKtot & $=$ & & 0.0000 & EPtot & $=$ & -135197.0665 \\
\hline BOND & $=$ & 1096.8230 & ANGLE & $=$ & & 47.0242 & DIHED & $=$ & 3541.2099 \\
\hline $1-4 \mathrm{NB}$ & $=$ & 1061.6433 & 1-4 EEL & $=$ & 117 & 90.9157 & VDWAALS & $=$ & 16209.837 \\
\hline EELEC & $=$ & -171344.5200 & EHBOND & $=$ & & 0.0000 & RESTRAINT & $=$ & 0.0000 \\
\hline EKCMT & $=$ & 0.0000 & VIRIAL & $=$ & & 0.0000 & $\begin{array}{l}\text { VOLUME } \\
\text { Density }\end{array}$ & $\begin{array}{l}= \\
=\end{array}$ & $\begin{array}{r}448410.716 \\
1.017\end{array}$ \\
\hline
\end{tabular}

$\mathrm{DV} / \mathrm{DL}=\quad-35.4512$

Ewald error estimate: $0.2740 \mathrm{E}-04$

\begin{tabular}{|c|c|c|c|c|c|c|}
\hline Softcore part & of the s) & stem: & 3 atoms, & TEMP (K) & $=$ & 215.86 \\
\hline SC_Etot $=$ & 18.2607 & SC_EKtot $=$ & 1.9303 & SC_EPtot & $=$ & 16.3304 \\
\hline SC_BOND= & 4.5587 & SC_ANGLE= & 1.7758 & SC_DIHED & $=$ & 9.9882 \\
\hline$S^{-} 14 N B=$ & 0.0088 & SC_14EEL $=$ & 0.0000 & SC_VDW & $=$ & -0.0012 \\
\hline SC_EEL = & 0.0000 & & & & & \\
\hline SC_RES_DIST $=$ & 0.0000 & SC_RES_ANG $=$ & 0.0000 & SC_RES_T & $5=$ & 0.0000 \\
\hline SC_EEL_DER= & 0.0000 & SC_VDW_DER= & 0.0000 & SC_DERIV & $=$ & 0.0000 \\
\hline
\end{tabular}

| TI region 2

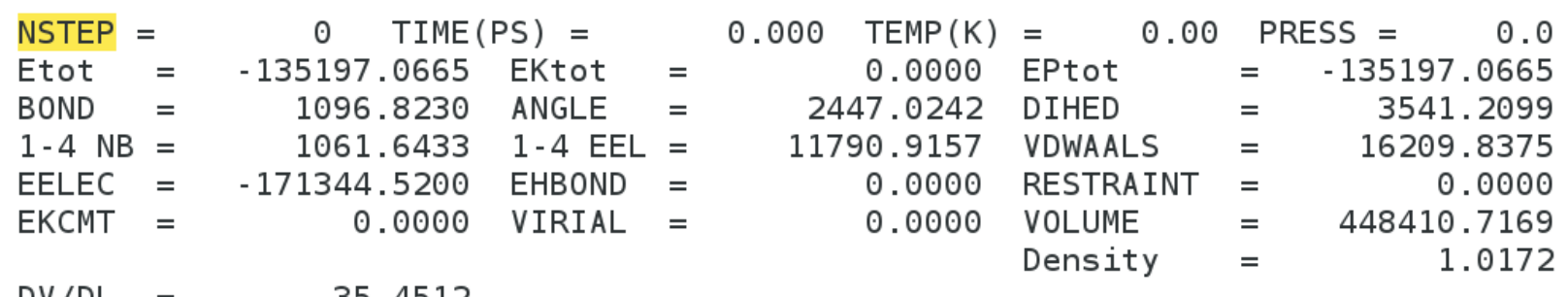

$\mathrm{DV} / \mathrm{DL}=\quad-35.4512$

Ewald error estimate: $0.2740 \mathrm{E}-04$

VDW CPU 
| TI region 1

\begin{tabular}{|c|c|c|c|c|c|c|c|c|c|}
\hline NSTEP & $=$ & TIME & PS ) & & 0.000 & TEMP (K) & 0.00 & & $\mathrm{SS}=$ \\
\hline Etot & $=$ & -135160.4071 & EKtot & $=$ & & 0.0000 & EPtot & $=$ & -135160.4071 \\
\hline BOND & $=$ & 1037.8846 & ANGLE & $=$ & 24 & 79.9287 & DIHED & $=$ & 3510.4997 \\
\hline $1-4 N B$ & $=$ & 1042.1221 & 1-4 EEL & $=$ & 118 & 51.8884 & VDWAALS & $=$ & 15884.17 \\
\hline EELEC & $=$ & - 170976.9085 & EHBOND & $=$ & & 0.0000 & RESTRAINT & $=$ & 0.000 \\
\hline EKCMT & $=$ & 0.0000 & VIRIAL & $=$ & & 0.0000 & $\begin{array}{l}\text { VOLUME } \\
\text { Density }\end{array}$ & $=$ & $\begin{array}{r}448671.130 \\
1.016\end{array}$ \\
\hline
\end{tabular}

$\mathrm{DV} / \mathrm{DL}=-0.4194$

Ewald error estimate: $0.1163 \mathrm{E}-03$

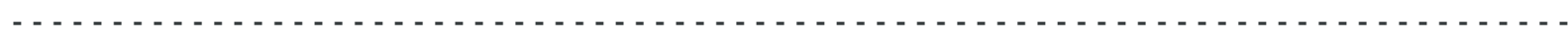

| TI region 2

\begin{tabular}{|c|c|c|c|c|c|c|c|c|c|}
\hline NSTEP & $=$ & TIME & PS) $=$ & & 0.000 & $\operatorname{TEMP}(\mathrm{K})$ & 0.00 & & ESS = \\
\hline Etot & $=$ & -135160.4071 & EKtot & $=$ & & 0.0000 & EPtot & $=$ & -135160.4071 \\
\hline BOND & $=$ & 1037.8846 & ANGLE & $=$ & 247 & 79.9287 & DIHED & $=$ & 3510.4997 \\
\hline $1-4 \mathrm{NB}$ & $3=$ & 1042.1221 & 1-4 EEL & $=$ & 1186 & 61.8884 & VDWAALS & $=$ & 15884.1778 \\
\hline EELEC & $=$ & - 170976.9085 & EHBOND & $=$ & & 0.0000 & RESTRAINT & $=$ & 0.0000 \\
\hline EKCMT & $=$ & 0.0000 & VIRIAL & $=$ & & 0.0000 & $\begin{array}{l}\text { VOLUME } \\
\text { Density }\end{array}$ & $\begin{array}{l}= \\
=\end{array}$ & $\begin{array}{r}448671.1309 \\
1.0167\end{array}$ \\
\hline $\begin{array}{l}\text { DV/DL } \\
\text { Ewald }\end{array}$ & $\begin{array}{l}= \\
\text { erro }\end{array}$ & $\begin{array}{l}-0.4194 \\
\text { estimate: }\end{array}$ & $0.1163 \mathrm{E}-\mathrm{C}$ & & & & & & \\
\hline
\end{tabular}

\section{Discharge CPU}

| TI region 1

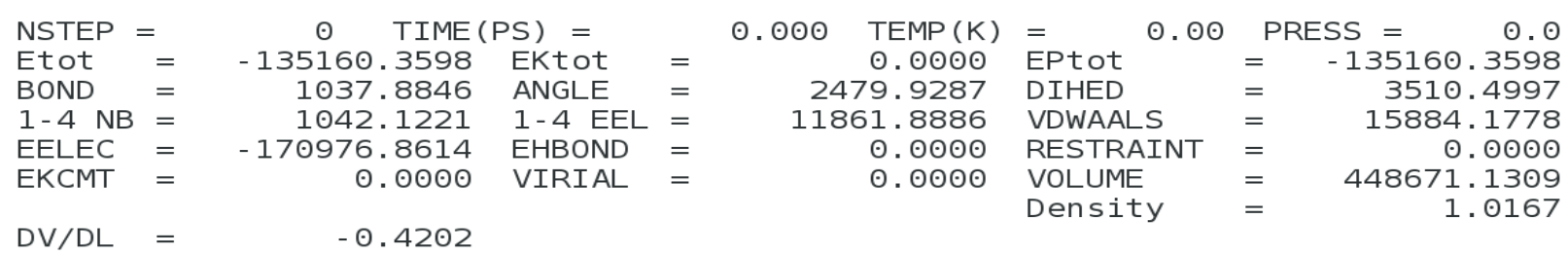

| TI region 2

\begin{tabular}{|c|c|c|c|c|c|c|c|c|c|}
\hline NSTEP & $=$ & $\odot \quad$ TIME & DS) = & & $\odot . \odot \odot \odot$ & $\operatorname{TEMP}(\mathrm{K})$ & $\odot . \odot \odot$ & & $E S S=\quad \odot . \odot$ \\
\hline Etot & $=$ & -135160.3598 & EKtot & $=$ & & $\odot . \odot \odot \odot \odot$ & EPtot & $=$ & -135160.3598 \\
\hline BOND & $=$ & 1037.8846 & ANGLE & $=$ & 247 & 9.9287 & DIHED & $=$ & 3510.4997 \\
\hline $1-4 N B$ & $=$ & 1042.1221 & 1-4 EEL & $=$ & 1186 & 1.8886 & VDWAALS & $=$ & 15884.1778 \\
\hline EELEC & $=$ & -170976.8614 & EHBOND & $=$ & & & RESTRAINT & $=$ & $\odot .0 \odot \odot \odot$ \\
\hline EKCMT & $=$ & $\odot . \odot \odot \odot \odot$ & VIRIAL & $=$ & & $\odot .0000$ & $\begin{array}{l}\text { VOLUME } \\
\text { Density }\end{array}$ & $\begin{array}{l}= \\
=\end{array}$ & $\begin{array}{r}448671.1309 \\
1.0167\end{array}$ \\
\hline $\mathrm{V} / \mathrm{DL}$ & $=$ & -0.4202 & & & & & & & \\
\hline
\end{tabular}

\section{Discharge GPU}

SI Figure 2: output initial energies for both Discharge and VDW changes in RFEB calculation. 